\title{
Efficacy of Acupuncture Treatment for Incidence of Poststroke Comorbidities: A Systematic Review and Meta-Analysis of Nationalized Cohort Studies
}

\author{
Li-Kung Wu ${ }^{D}$, ${ }^{1}$ Chung-Shan Hung, ${ }^{3,4}$ Yen-Lun Kung, ${ }^{1,2}$ Zhong-Kui Chen, ${ }^{1,5}$ \\ Shinn-Zong Lin $\left(\mathbb{1},{ }^{6,7}\right.$ Jaung-Geng Lin $\left(\mathbb{1},{ }^{8,9}\right.$ and Tsung-Jung $\mathrm{Ho}\left(\mathbb{C}^{1,2,5}\right.$ \\ ${ }^{1}$ Department of Chinese Medicine, Hualien Tzu Chi Hospital, Buddhist Tzu Chi Medical Foundation, Hualien, Taiwan \\ ${ }^{2}$ School of Post-Baccalaureate Chinese Medicine, Tzu Chi University, Hualien, Taiwan \\ ${ }^{3}$ Department of Aging and Community Health, Hualien Tzu Chi Hospital, Buddhist Tzu Chi Medical Foundation, \\ Hualien, Taiwan \\ ${ }^{4}$ Department of Public Health, Tzu Chi University, Hualien, Taiwan \\ ${ }^{5}$ Integration Center of Traditional Chinese and Modern Medicine, Hualien Tzu Chi Hospital, Hualien, Taiwan \\ ${ }^{6}$ Bioinnovation Center, Tzu Chi Foundation, Department of Neurosurgery, Hualien Tzu Chi Hospital, \\ Buddhist Tzu Chi Medical Foundation, Tzu Chi University, Hualien, Taiwan \\ ${ }^{7}$ Department of Neurosurgery, Hualien Tzu Chi Hospital, Buddhist Tzu Chi Medical Foundation, Hualien, Taiwan \\ ${ }^{8}$ Research Center for Chinese Medicine \& Acupuncture, China Medical University, Taichung, Taiwan \\ ${ }^{9}$ School of Chinese Medicine, China Medical University, Taichung, Taiwan
}

Correspondence should be addressed to Jaung-Geng Lin; jglin@mail.cmu.edu.tw and Tsung-Jung Ho; jeron888@gmail.com

Received 4 July 2021; Revised 12 December 2021; Accepted 20 December 2021; Published 1 February 2022

Academic Editor: Mozaniel Oliveira

Copyright (c) $2022 \mathrm{Li}$-Kung Wu et al. This is an open access article distributed under the Creative Commons Attribution License, which permits unrestricted use, distribution, and reproduction in any medium, provided the original work is properly cited.

Acupuncture has been applied as a complementary therapy in stroke survivors worldwide and approved to be beneficial to stroke recovery. However, there is little medical evidence regarding the association between acupuncture and the risk of poststroke comorbidities. We reviewed big data studies from the Taiwan National Health Insurance Research Database to investigate the risk of poststroke comorbidities after acupuncture treatment in a real-world situation. Ten English (PubMed, Embase, Medline, Cochrane, Alt HealthWatch, CINAHL, Health Source, PsycINFO, PsycARTICLES, and Psychology and Behavioral Sciences Collection) and two Chinese (AiritiLibray and Visualizing Health Data) electronic databases were searched from inception until December 2020 for nationalized cohort studies comparing the effects of acupuncture treatment with a nonacupuncture control group among stroke patients. Eight nationalized cohort studies were included. Six of eight studies showed a moderate overall risk of bias, while two studies showed a serious overall risk of bias. Included studies have investigated the effect of acupuncture in reducing the risk of seven medical conditions after stroke, including stroke recurrence, new-onset acute myocardial infarction (AMI), pneumonia, dementia, epilepsy, urinary tract infection (UTI), and depression. The meta-analysis showed clinically significant reductions in the risk of poststroke comorbidities in the acupuncture group compared to the nonacupuncture group (HR, 0.776; 95\% CI, 0.719-0.838; $p<0.0001$ ). In this systematic review and meta-analysis of nationalized cohort studies, acupuncture showed clinically relevant benefits in reducing the incidence of poststroke comorbidities, such as stroke recurrence, new-onset acute myocardial infarction (AMI), pneumonia, dementia, epilepsy, and UTI.

\section{Introduction}

Stroke is the second leading cause of death and a major cause of acquired disability worldwide [1]. Among stroke patients, acupuncture has been practiced in traditional Chinese medicine for thousands of years and applied as a complementary therapy in stroke survivors [2]. Acupuncture has been authenticated to improve motor impairment [3], alleviate neurological deficiency [4], reduce psychological symptoms [5], increase local blood circulation [6], and 
modulate immunology [7] for the stroke patient. Nevertheless, comorbidities and complications of stroke have a huge impact on the prognosis of stroke $[8,9]$. Stroke patients display a higher susceptibility to neurological disorders (such as seizure, epilepsy, dementia, and cognitive impairment), infectious diseases (pneumonia and urinary tract infection, UTI), cardiovascular diseases (deep venous thrombosis and acute myocardial infarction, AMI), psychological disorders (depression), and secondary stroke [10]. However, there is little medical evidence regarding the association between acupuncture and the risk of poststroke comorbidities.

Under National Health Insurance coverage, people in Taiwan can receive 15 acupuncture treatments per month in a hospital or at a clinic after stroke. With inexpensive cost and without obvious adverse events [11], acupuncture in stroke patients is well accepted and gradually increasing in Taiwan [2].

Although well-designed randomized control trials (RCT) have proved the efficacy of acupuncture for stroke recovery [12], the limitations, such as small sample size, difficulty in blinding of acupuncture, risks of bias due to deviations from intended interventions, and a lack of longterm follow-up, may negatively influence the reliability and generalization of the outcome.

Moreover, the rigorous-designed clinical trial differs from real-world situations. In clinical practice, acupuncture was conducted by traditional Chinese doctors with diversified and individualized manipulation and acupoint selection. Additionally, in a busy clinical setting, it may be impossible for traditional Chinese doctors to follow the rigorous protocols of RCTs. Traditional Chinese doctors seldom treat specific diagnoses or symptoms but provide holistic intervention that considers the balance of the whole person. Despite the same symptoms, traditional Chinese medical treatment may differ between patients with different physical constitutions. Therefore, the well-established protocol of RCT is not applicable to clinical traditional Chinese medicine practice.

However, investigation of the effect of clinical traditional Chinese treatment is indispensable for both doctors and patients. The cohort study is the best study design that can provide evidence-based information of real-world clinical practice with a long-term prognosis and a large population. In Taiwan, the Taiwan National Health Insurance Research Database (NHIRD) provides anonymous medical records of all insured individuals and was used by numerous cohort studies to reveal clinical practice behavior. To investigate the risk of poststroke comorbidities after acupuncture treatment in a real-world situation, we conducted a systematic review of nationwide cohort studies that have used big data from the NHIRD.

\section{Methods}

2.1. Search Strategy. Ten English electronic databases (PubMed, Embase, Medline, Cochrane, Alt HealthWatch, CINAHL, Health Source, PsycINFO, PsycARTICLES, and Psychology and Behavioral Sciences Collection) and two
Chinese electronic databases (AiritiLibray and Visualizing Health Data) were searched from inception until December 2020. Key search queries were (Stroke OR Cerebrovascular disorder OR Cerebrovascular Accident OR cerebrovascular disease OR cerebral ischemia OR ischemic stroke OR hemorrhagic stroke OR brain infarction OR cerebral infarction OR CVA) AND (Acupuncture OR Electroacupuncture OR electro-acupuncture OR Auricular Acupuncture OR Ear Acupuncture OR Scalp Acupuncture OR Acupuncture Point OR Acupoint) AND (NHIRD OR National Health OR Health Insurance OR Nation*). Reference lists of full-text papers were searched. Relevant studies were also screened. Figure 1 shows the systematic literature search performed by two authors (Wu and Hung).

\subsection{Inclusion and Exclusion Criteria. Included studies met} the following criteria. (1) Taiwan's National Health Insurance Research Database, (2) participants of the study were diagnosed with stroke by the International Classification of Diseases, 9th Revision, Clinical Modification codes (ICD-9CM 430-437), (3) the study compared acupuncture with nonacupuncture control groups, and (4) the study showed changes in poststroke comorbidities or medical outcome. Other interventions, such as traditional Chinese medicine, laser acupuncture, acupressure, and massage, were excluded. Studies that did not evaluate acupuncture for stroke-related medical outcomes in NHIRD were excluded.

2.3. Data Abstraction and Rating of Articles for ROB. Study selection, data extraction, risk of bias assessment, and quality evaluation were performed independently by two authors ( $\mathrm{Wu}$ and Hung). The condition, trial sample size, study duration, outcome incidence, between-group hazard ratio, and log-rank test were extracted from selected studies. Two authors rated articles for the confounding, selection of participants, classification of interventions, deviations from intended interventions, missing data, measurement of outcomes, selection of the reported result, and overall bias via the assessment tool for ROBINS-I [13] from the Cochrane Handbook for Systematic Reviews of Interventions $[14,15]$. Rating differences were settled by discussion or by a third person (Ho) if consensus was not achieved.

2.4. Data Analysis. Data were summarized using hazard ratios (HR) with 95\% CIs for binary outcomes or mean difference (MD) with a 95\% CI for continuous outcomes. We used R [16] and the package meta [17] for meta-analysis if the trials had acceptable homogeneity of study design, participants, interventions, controls, and outcome measures. Statistical heterogeneity was tested by examining $I^{2}$ or $p$-value; an $I^{2}>50 \%$ or a $p$-value $<0.1$ indicates the possibility of statistical heterogeneity [18]. Both a fixed-effect model and a random-effects model were used if there was a possibility of statistical heterogeneity among the trials. If the $I^{2}$ was $<50 \%$ or the $p$-value was $>0.1$, only a fixed-effect model was used for meta-analysis. 


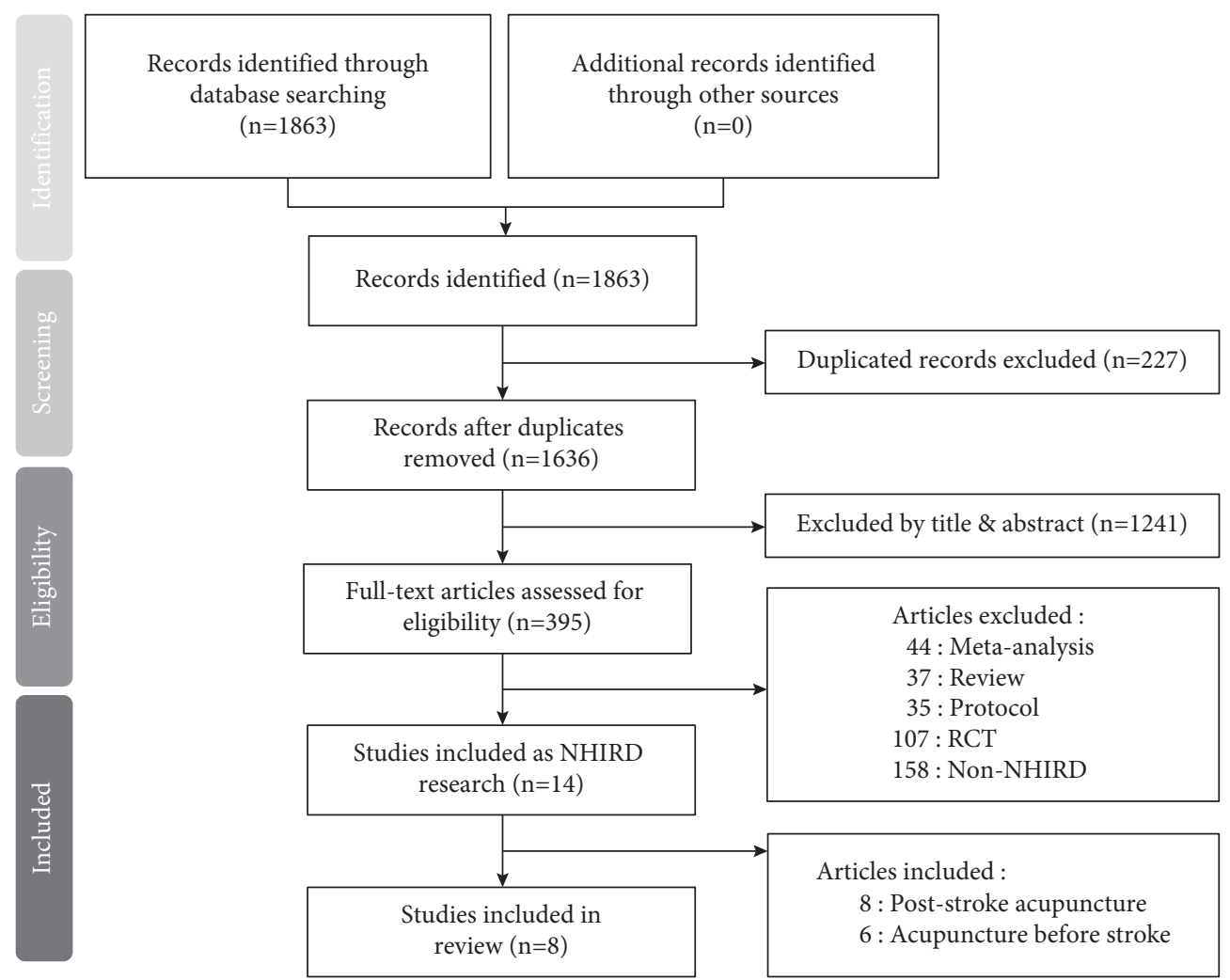

FIGURE 1: Preferred reporting items for systematic reviews and meta-analyses (PRISMA) guidelines indicating parameters for the search and identification of included studies.

\section{Results}

3.1. Study Search and Characteristics. Our search strategy retrieved 1,863 potentially eligible articles (Figure 1). A total of 227 duplicates were eliminated. Additional records (1,241) were also excluded because their titles and abstracts were not related to acupuncture, stroke, or Taiwan's NHIRD. After an assessment of full-text articles, 381 records were excluded because of the irrelevance of NHIRD and different study designs. Since the present review emphasized poststroke medical conditions, 6 articles were excluded because the acupuncture treatments were used before the cerebrovascular accident. Eventually, 8 articles were included in our systematic review.

3.2. Quality Assessment. The risk of bias of all included studies was assessed using ROBINS-I [13]. Six of eight studies were rated with moderate bias risk due to limitations in study design regarding confounder control (Figure 2 ). The NHIRD could not control the confounders, such as BMI, the severity of the stroke, family history, education level, cognitive function, mobility function, and so on. However, the six studies have controlled all confounders that could be controlled. Two studies were rated with a serious risk of confounding bias due to uncontrolled known confounders. Yang et al. [19] investigated the risk of UTI without controlling the usage of a bladder catheter. Lu et al. [20] investigated the incidence of depression after acupuncture without controlling the acupuncture sections after the acute stage. Owing to the nature of NHIRD studies that analyze the records from a big database, the risks of classification, deviations from intended interventions, missing data, and the measurement of outcomes were low in all studies. The outcomes of the NHIRD were the risk or incidence of specific diagnosis after the intervention. Therefore, the risk of bias in outcome measurements was low in NHIRD studies. The risk of bias in the selection of the reported results was moderate in all studies because there is no preregistered system for the NHIRD or big data studies. To summarize, six of eight studies were categorized with a moderate overall risk of bias due to the limitation of study design in confounder control and selection of the reported results [21-26]. Two studies were categorized with a serious overall risk of bias due to failure to control the known and controllable confounders [19, 20].

3.3. Outcomes. Studies have investigated the effect of acupuncture in reducing the risk of seven medical conditions after stroke, including six studies involving stroke recurrence, newonset AMI, pneumonia, dementia, epilepsy, and UTI, and two studies involving poststroke depression (Table 1). Compared to stroke patients who did not receive acupuncture, stroke patients who had received acupuncture displayed a lower incidence of stroke recurrence in ischemic stroke (HR: 0.88; 95\% CI: 0.84-0.91), lower incidences of new-onset AMI (HR: 0.86; 95\% CI: 0.80-0.93), pneumonia (HR: 0.86; 95\% CI: 0.82-0.90), dementia (HR: 0.73; 95\% CI: 0.66-0.80), epilepsy (HR: 0.74; 95\% CI: 0.68-0.80), and UTI (HR: 0.76; 95\% CI: 0.73-0.80). 
Risk of bias domains
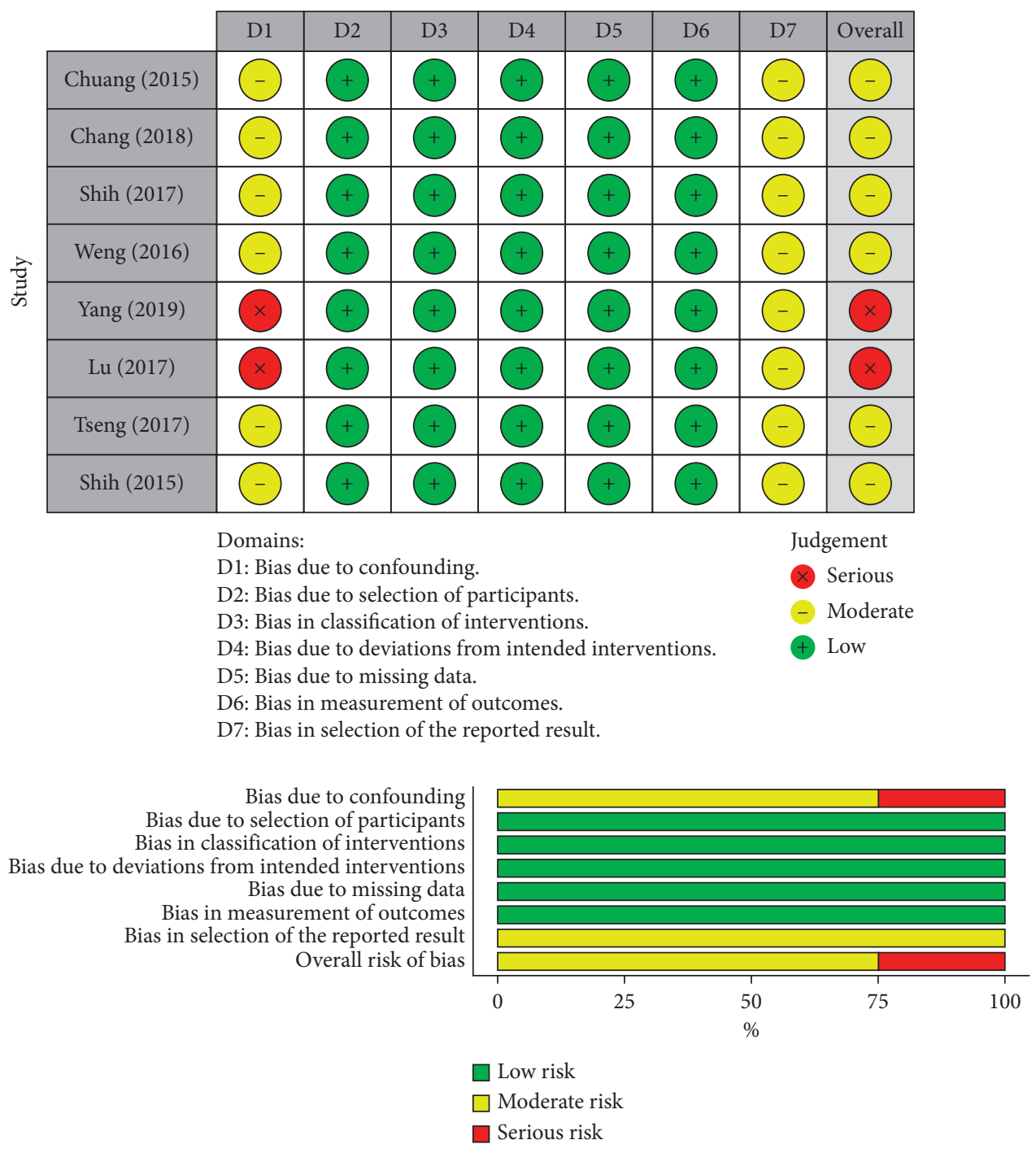

FIGURE 2: Cochrane risk of bias in nonrandomized studies of interventions (ROBINS-I) for included studies.

The association of acupuncture and poststroke depression was inconsistent in the two studies. Tseng et al. indicated the reduced risk of poststroke depression with HR of 0.48 (95\% CI: 0.39-0.58) and 0.72 (95\% CI: 0.61-0.84) for patients who received frequent and infrequent acupuncture treatments, respectively. Conversely, Lu et al. reported an HR of 1.04 (95\% CI: 0.84-1.29) The meta-analysis result of the two studies was provided in the Supplemental Figure S1, the result showed a HR of 0.70 (95\% CI: 0.47-1.05) favors acupuncture group but without significant difference.. However, it should be noted that $\mathrm{Lu}$ et al. defined stroke patients who received any acupuncture therapies within three months of the discharge as acupuncture users. This definition of acupuncture users may include patients who did not receive enough dosage of acupuncture intervention for observable changes, may exclude patients who received acupuncture after three months of discharge, and may result in an underestimation of the effects of acupuncture treatment.

The pooling analysis of eight trials showed that acupuncture treatment significantly reduced the risk of poststroke comorbidities compared to the nonacupuncture group in a random-effects model (HR, 0.776; 95\% CI, $0.719-0.838 ; p<0.0001)$ with significant heterogeneity $\left(I^{2}=89.2 \%\right.$; 95\% CI, 81.7\%-93.6\%). The forest plot of metaanalysis results is shown in Figure 3 (Supporting data for forest plot are provided in the Supplemental Table S1). The results from Tseng et al. were separated into two trials (frequent and infrequent acupuncture users compared with acupuncture nonusers) in the pooling analysis.

3.4. Subgroup Analysis. The extraction data of subgroup analysis are shown in Table 2. The effects of acupuncture on acute myocardial infarction, pneumonia, dementia, epilepsy, UTI, and stroke recurrence risk were significant in both genders, with a tendency toward favoring the female gender $[19,21,23-26]$. However, the results are controversial regarding poststroke depression $[20,25]$.

In the subgroup analysis of different types of strokes, our review indicated the positive influence of acupuncture 


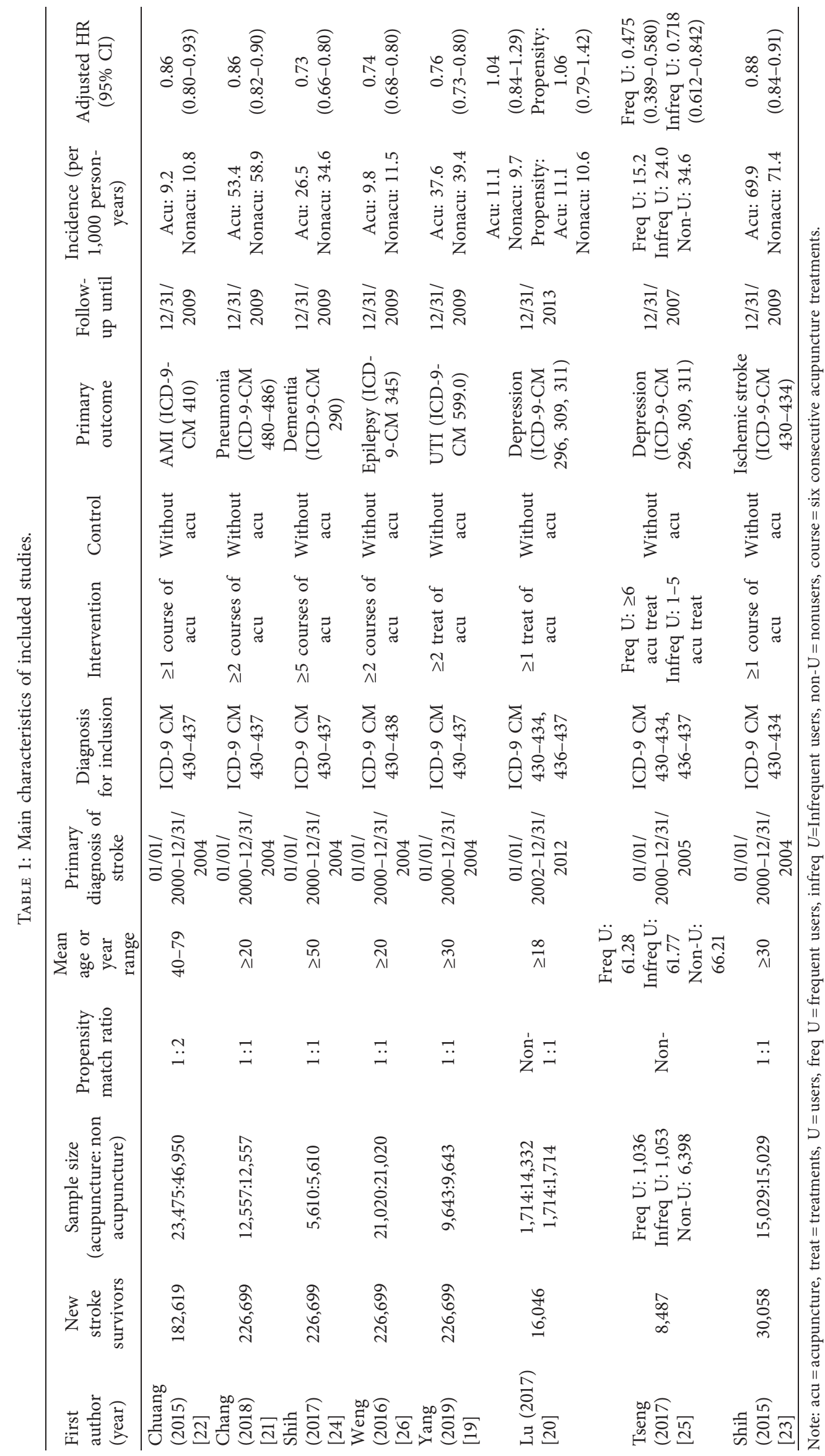




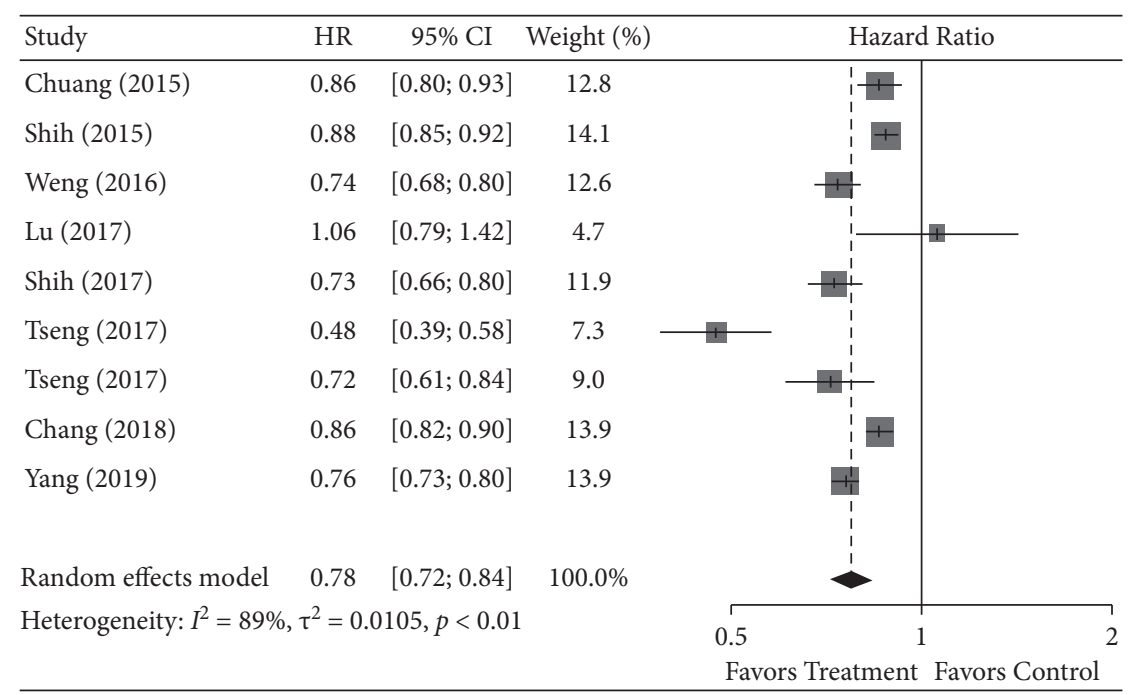

FIGURE 3: Forest plot of hazard ratio (HR) of poststroke comorbidities with acupuncture intervention compared with nonacupuncture control.

therapy in reducing the risk of pneumonia, epilepsy, and UTI in patients with all stroke subtypes, including hemorrhagic, ischemic, and other stroke types [19, 21, 26].

In the subgroup analysis of the number of medical conditions, patients with more medical conditions had higher hazard ratios for poststroke pneumonia, dementia, UTI, depression, and stroke recurrence [19, 21, 23-25].

In different age strata, the younger population displayed lower HRs after acupuncture treatment for poststroke pneumonia, epilepsy, and stroke recurrence. In the metaanalysis of six included studies with age strata [19, 21-24, 26] (Supplemental Table S2), acupuncture treatment showed an age-dependent increase in HR in overall comorbidities after stroke (Figure 4) (Supplemental Table S3).

The number of acupuncture treatments also plays a crucial role in the incidence of poststroke comorbidities. The risk of acute myocardial infarction [22], pneumonia [21], epilepsy [26], UTI [19], and stroke recurrence [23] exhibited a dose-dependent decrease with increasing sessions of acupuncture treatment (Supplemental Table S4). The metaanalysis of the above five included studies showed a lower risk for overall poststroke comorbidities as the number of acupuncture treatment packages increased (Figure 5) (Supplemental Table S5). Tseng et al. showed the probability of poststroke depression in patients was reduced with frequent acupuncture (HR: $0.475(0.389-0.580)$ ) and infrequent acupuncture (HR $0.718(0.612-0.842)$ ), compared to those not receiving acupuncture treatments [25].

To summarize, the effects of acupuncture treatment on poststroke comorbidities are associated with gender, the number of medical conditions, age, and the number of acupuncture treatments received.

\section{Discussion}

Regarding the limitations of RCT that cannot reflect the real effect of clinical practice, this review identified eight NHIRD studies involving acupuncture for poststroke comorbidities. The results indicated that patients who had received acupuncture were at lower risk of poststroke comorbidities, including AMI, pneumonia, dementia, epilepsy, UTI, and stroke recurrence. To our knowledge, the review is the first study to summarize the results of big data analyses from NHIRD. NHIRD studies with nationalized records of acupuncture that cumulated from real-world practice can provide the most direct evidence for clinical use without excluding the diversity and individualization of the traditional Chinese intervention from study procedures.

4.1. Evidence-Based Medicine for Acupuncture in the Treatment of Stroke Patients. Many empirical studies investigated the effects of acupuncture on comorbidities after stroke. In studies related to poststroke pneumonia, acupuncture is effective in decreasing the incidence and morbidity of pneumonia in patients with poststroke syndrome [27, 28]. For urology, acupuncture was an effective treatment for poststroke urodynamic detrusor overactivity [29]. Electroacupuncture also showed beneficial effects on stroke survivors with incomplete bladder emptying, thus improving urinary function [30]. Besides, acupuncture showed a clinically relevant decrease in stroke relapse compared with sham acupuncture [31]. For poststroke insomnia, a metaanalysis showed that acupuncture appeared to be more effective than conventional drugs [32]. For vascular dementia, scalp acupuncture could improve the clinical intelligence level of dementia induced by cerebral infarction [33]. For poststroke depression, filiform needle acupuncture can reduce the Hamilton Depression Rating Scale (HAMD) level compared to anti-depressant drugs after treatment for two weeks [5]. Another systematic review and meta-analysis also indicated that acupuncture shows greater effects on poststroke depression, with a better safety profile than antidepressants [34]. Overall, for poststroke comorbidities, acupuncture showed positive effects on pneumonia, urinary function, stroke relapse, insomnia, vascular dementia, and depression in stroke survivors in rigorous-designed experimental environments. 
TABLE 2: Risks of poststroke comorbidities associated with acupuncture treatment in patients with stroke stratified by sex, type of stroke, medical conditions, and age.

\begin{tabular}{|c|c|c|c|c|}
\hline Author (year) & Gender & Types of stroke & Baseline medical conditions & Age strata \\
\hline Chuang (2015) [22] & $\begin{array}{c}\text { Female HR: } 0.85^{*} \\
(0.76-0.95) \\
\text { Male HR: } 0.87^{*} \\
(0.80-0.95)\end{array}$ & $\begin{array}{l}\text { Hemorrhagic HR: } \\
0.62^{*}(0.44-0.88) \\
\text { Ischemic HR: } \\
0.87^{*}(0.79-0.95) \\
\text { Others HR: } 0.89 \\
(0.79-1.01)\end{array}$ & Nil & $\begin{array}{c}40-49 \text { HR: } 0.84(0.62-1.14) \\
50-59 \text { HR: } 0.75^{*}(0.63-0.90) \\
60-69 \text { HR: } 0.85^{*}(0.75-0.95) \\
70-79 \text { HR: } 0.93(0.83-1.03)\end{array}$ \\
\hline Chang (2018) [21] & $\begin{array}{l}\text { Female HR: } 0.79^{*} \\
(0.70-0.82) \\
\text { Male HR: } 0.92^{*} \\
(0.86-0.98)\end{array}$ & $\begin{array}{c}\text { Hemorrhagic HR: } 0.66^{*} \\
(0.53-0.81) \\
\text { Ischemic HR: } 0.86^{*} \\
(0.81-0.92) \\
\text { Others HR: } 0.87^{*} \\
(0.80-0.95)\end{array}$ & $\begin{array}{l}0 \text { HR: } 0.49^{*}(0.43-0.57) \\
1 \text { HR: } 0.77^{*}(0.70-0.85) \\
2 \text { HR: } 0.92(0.84-1.01) \\
3 \text { HR: } 1.10(1.01-1.19)\end{array}$ & $\begin{array}{l}20-29 \text { HR: } 0.16^{*}(0.04-0.69) \\
30-39 \text { HR: } 0.52^{*}(0.28-0.98) \\
40-49 \text { HR: } 0.73^{*}(0.58-0.93) \\
50-59 \text { HR: } 0.83^{*}(0.72-0.95) \\
60-69 \text { HR: } 0.82^{*}(0.75-0.89) \\
70-79 \text { HR: } 0.88^{*}(0.81-0.95) \\
\geq 80 \text { HR: } 0.86(0.73-1.00)\end{array}$ \\
\hline Shih (2017) [24] & $\begin{array}{l}\text { Female HR: } 0.70^{*} \\
(0.60-0.80) \\
\text { Male HR: } 0.75^{*} \\
(0.66-0.85)\end{array}$ & $\begin{array}{c}\text { Hemorrhagic HR: } 0.77 \\
(0.53-1.12) \\
\text { Ischemic HR: } 0.74^{*} \\
(0.66-0.84) \\
\text { Others HR: } 0.69^{*} \\
(0.57-0.82)\end{array}$ & $\begin{array}{l}0 \text { HR: } 0.55^{*}(0.39-0.77) \\
1 \text { HR: } 0.64^{*}(0.52-0.80) \\
2 \text { HR: } 0.81^{*}(0.68-0.98) \\
\geq 3 \text { HR: } 0.82^{*}(0.71-0.95)\end{array}$ & $\begin{array}{l}\text { 50-59 HR: } 0.64^{*}(0.47-0.86) \\
60-69 \text { HR: } 0.75^{*}(0.64-0.88) \\
70-79 \text { HR: } 0.72^{*}(0.63-0.83) \\
\geq 80 \text { HR: } 0.54^{*}(0.38-0.76)\end{array}$ \\
\hline Weng (2016) [26] & $\begin{array}{l}\text { Female HR: } 0.70^{*} \\
(0.61-0.81) \\
\text { Male HR: } 0.77^{*} \\
(0.69-0.85)\end{array}$ & $\begin{array}{c}\text { Hemorrhagic HR: } 0.60^{*} \\
(0.50-0.73) \\
\text { Ischemic HR: } 0.86^{*} \\
(0.78-0.96) \\
\text { Others HR: } 0.62^{*} \\
(0.52-0.74)\end{array}$ & Nil & $\begin{array}{c}20-29 \text { HR: } 0.16^{*}(0.04-0.68) \\
30-39 \text { HR: } 0.39^{*}(0.21-0.73) \\
40-49 \text { HR: } 0.51^{*}(0.39-0.66) \\
\text { 50-59 HR: } 0.66^{*}(0.54-0.80) \\
\text { 60-69 HR: } 0.79^{*}(0.68-0.91) \\
70-79 \text { HR: } 0.88(0.76-1.02) \\
\geq 80 \text { HR: } 0.71(0.48-1.04)\end{array}$ \\
\hline Yang (2019) [19] & $\begin{array}{l}\text { Female HR: } 0.73^{*} \\
(0.69-0.78) \\
\text { Male HR: } 0.80^{*} \\
(0.75-0.85)\end{array}$ & $\begin{array}{c}\text { SAH HR: } 0.58^{*} \\
(0.50-0.69) \\
\text { Ischemic HR: } 0.78^{*} \\
(0.74-0.83) \\
\text { TIA HR: } 0.81^{*} \\
(0.72-0.92) \\
\text { Others HR: } 0.72^{*} \\
(0.63-0.82)\end{array}$ & $\begin{array}{l}0 \text { HR: } 0.52^{*}(0.47-0.58) \\
1 \text { HR: } 0.74^{*}(0.69-0.80) \\
2 \text { HR: } 0.95(0.87-1.03) \\
\geq 3 \text { HR: } 0.91(0.83-1.01)\end{array}$ & $\begin{array}{c}\text { 30-39 HR: } 0.62(0.34-1.16) \\
40-49 \text { HR: } 0.65^{*}(0.53-0.79) \\
50-59 \text { HR: } 0.82^{*}(0.73-0.93) \\
60-69 \text { HR: } 0.74^{*}(0.69-0.80) \\
\geq 70 \text { HR: } 0.77^{*}(0.72-0.82)\end{array}$ \\
\hline $\mathrm{Lu}(2017)[20]$ & $\begin{array}{c}\text { Female HR: } 1.30 \\
(0.90-1.86) \\
\text { Male HR: } 1.05 \\
(0.76-1.45)\end{array}$ & $\begin{array}{c}\text { Hemorrhagic HR: } 0.70 \\
(0.42-1.16) \\
\text { Ischemic HR: } 1.12 \\
(0.88-1.43)\end{array}$ & Nil & $\begin{array}{l}<65 \text { HR: } 1.19(0.84-1.68) \\
\geq 65 \text { HR: } 1.07(0.77-1.50)\end{array}$ \\
\hline Tseng (2017) [25] & $\begin{array}{c}\text { Female HR: } 0.78 \\
(0.71-0.86) \\
\text { *Compared to male }\end{array}$ & $\begin{array}{c}\text { Hemorrhagic HR: } 0.80^{*} \\
(0.68-0.94) \\
\text { Ischemic HR: } 0.91 \\
(0.81-1.03) \\
{ }^{*} \text { Compared to other } \\
\text { types of stroke }\end{array}$ & $\begin{array}{c}\text { MI HR: } 1.30(1.08-1.56) \\
\text { Cancer HR: } 1.42(1.27-1.60) \\
\text { DM HR: } 1.14(1.03-1.25) \\
\text { CKD HR: } 1.52(1.35-1.72) \\
\text { COPD HR: } 1.22(1.10-1.36) \\
\text { THI HR: } 1.63(1.34-1.99) \\
\text { *Compared to patients without } \\
\text { the medical condition }\end{array}$ & $\begin{array}{c}\text { Older HR: } 1.045 \\
(1.040-1.050) \\
\text { *Compared to younger }\end{array}$ \\
\hline Shih (2015) [23] & $\begin{array}{l}\text { Female HR: } 0.83^{*} \\
(0.79-0.89) \\
\text { Male HR: } 0.91^{*} \\
(0.86-0.95)\end{array}$ & $\begin{array}{c}\text { Ischemic HR: } 0.88^{*} \\
(0.84-0.91)\end{array}$ & $\begin{array}{l}\text { HTN no HR: } 0.67^{*}(0.62-0.74) \\
\text { HTN yes HR: } 0.94^{*}(0.90-0.98) \\
\text { HPL no HR: } 0.84^{*}(0.81-0.88) \\
\text { HPL yes HR: } 0.97(0.90-1.04) \\
\text { DM no HR: } 0.83^{*}(0.79-0.87) \\
\text { DM yes HR: } 0.96(0.90-1.02)\end{array}$ & $\begin{array}{c}30-39 \text { HR: } 0.35^{*}(0.22-0.54) \\
40-49 \text { HR: } 0.61^{*}(0.52-0.71) \\
\text { 50-59 HR: } 0.81^{*}(0.74-0.89) \\
\text { 60-69 HR: } 0.85^{*}(0.80-0.91) \\
70-79 \text { HR: } 0.99(0.92-1.06) \\
\geq 80 \text { HR: } 1.15(0.98-1.34)\end{array}$ \\
\hline
\end{tabular}

Note: $\mathrm{TIA}=$ transient ischemic attack, $\mathrm{SAH}=$ subarachnoid hemorrhage, $\mathrm{MI}=$ myocardial infarction, $\mathrm{CKD}=$ chronic kidney diseases, $\mathrm{COPD}=$ chronic obstructive pulmonary disease, $\mathrm{THI}=$ traumatic head injury, $\mathrm{DM}=$ diabetes mellitus, $\mathrm{HTN}=$ hypertension, and $\mathrm{HPL}=$ hyperlipidemia. ${ }^{*}$ Significant difference. 


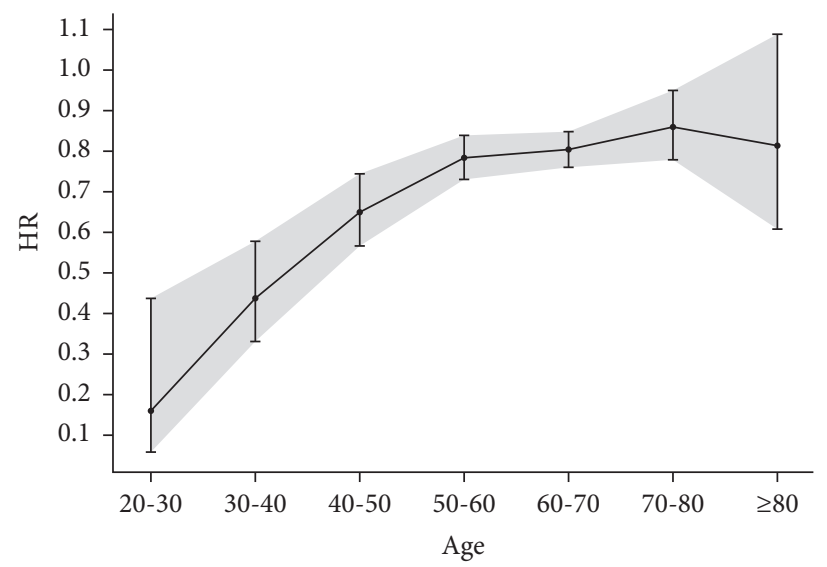

Figure 4: Meta-analysis results showing HRs with 95\% confidence intervals of different age strata on poststroke comorbidities after acupuncture treatment.

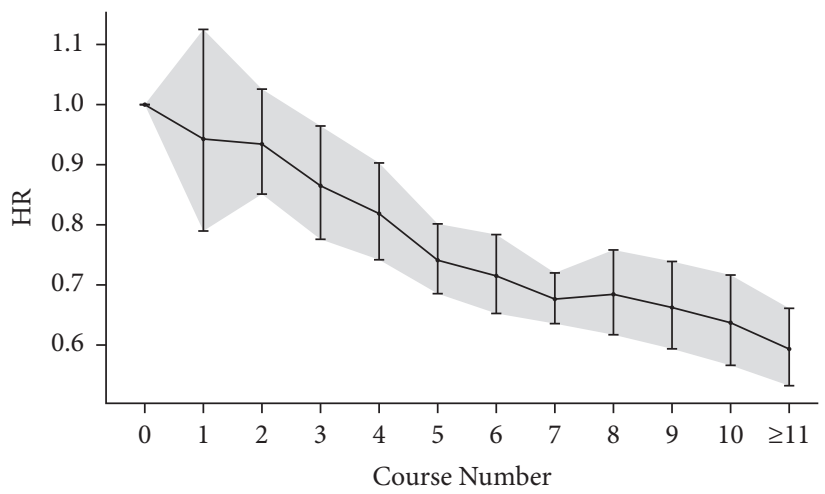

FIgURE 5: Meta-analysis results showing HRs with $95 \%$ confidence intervals of various acupuncture courses on poststroke comorbidities after acupuncture treatment.

In addition to the effects on poststroke comorbidities, acupuncture has proven effects on symptoms, sequelae, and functions in a well-controlled experimental environment regarding evidence of RCT, systematic review, and metaanalysis $[35,36]$. Acupuncture effectively reduces neurological impairments [11,37], motor impairments [3, 38-40], dysphagia [37, 41], and other symptoms, such as fatigue [42], insomnia [32], and pain [43]. Acupuncture also assists with cognitive function [4] and activities of daily living $[3,39]$ after stroke. Moreover, acupuncture increases mean flow velocity in both hemispheres [6], displays protective effects on brain reperfusion injury [44], and improves neurogenesis [45].

4.2. Mechanism of Acupuncture for Poststroke Comorbidities. The mechanism behind how acupuncture reduces the incidence of poststroke comorbidities is still unclear. However, there are several possible underlying mechanisms, including immunological, neurological, hemodynamic, neuroendocrine, and metabolic mechanisms.

Regarding the immunological mechanism, acupuncture can directly modulate specific and nonspecific immunity to correct systemic immunosuppression and decrease susceptibility to infection after stroke [46]. Acupuncture decreases certain cytokines and inhibits Th1 cell responses to regulate Th1/Th2 balance $[47,48]$, which circumvent infectious diseases [49]. Furthermore, acupuncture is related to systemic anti-inflammatory responses, which are mediated by reducing endotoxin-induced inflammation [50], decreasing whole blood tumor necrosis factor- $\alpha$ (TNF- $\alpha$ ) [51], and upregulating IL-10 production [52]. These studies demonstrate that acupuncture may contribute to modulating immune system balance, reducing the inflammatory response, and correcting systemic immunosuppression and may lead to improvements in poststroke UTI and pneumonia incidences [46, 49].

In the neurological mechanism, acupuncture can stimulate multiple brain networks and regulate functional connectivity by activating under-activated brain regions while deactivating overloaded areas [53], which may relate to how acupuncture alleviates incidences of epilepsy and dementia. Moreover, in terms of the autonomic nerve system, the autonomic shift may play an important role in increased susceptibility to infections after intracerebral hemorrhage. Acupuncture regulates the activities of the autonomic nervous system by enhancing vagal nerve activity and decreasing sympathetic nerve activity [54]. By correcting 
autonomic shifts, acupuncture may reduce the susceptibility of infections and decrease the incidence of UTI and pneumonia.

Regarding the hemodynamic mechanism, acupuncture can increase cerebral blood flow $[6,55]$, enhance local blood perfusion [56], promote angiogenesis [57], and lower blood pressure [58]. Previous studies demonstrated that acupuncture increased nitric oxide generation, which could regulate local blood circulation and relieve atherosclerosis development [56]. These hemodynamics effects may contribute to reducing risks of AMI and recurrent stroke [59].

In the neuroendocrine mechanism, acupuncture therapy upregulates the expression of serotonin $1_{\mathrm{A}}$ receptor $\left(5-\mathrm{HT} 1_{\mathrm{A}}\right.$ receptor) in the cortex, thalamus, hippocampus, and the hypothalamus, which contribute to alleviating the depressive-like behavior [60]. In addition, acupuncture effectively cures chronic pain [61]. Through regulation of the serotonin receptor and pain reduction, acupuncture may alleviate the causes of depression.

In the metabolic mechanism, acupuncture treatment can reduce serum triglycerides, low-density lipoprotein, cholesterol, and total cholesterol levels [62] and is consequently effective in reducing the risk of AMI and recurrent stroke [63].

In addition to the immunological, neurological, hemodynamic, neuroendocrine, and metabolic mechanisms, other indirect factors may also enhance the effects of acupuncture on poststroke comorbidities. For example, a previous study showed that acupuncture significantly decreases residual urine and may decrease the incidence of UTI [64]. In addition, improved motor ability induced by acupuncture $[65,66]$ may contribute to better physical mobility, more aerobic activities, and improved cardiopulmonary fitness associated with reduced risks of AMI and recurrent stroke $[67,68]$.

4.3. Subgroup Analysis. According to subgroup analysis results, the effects of acupuncture on poststroke comorbidities were associated with gender, the number of medical conditions, age, and the number of acupuncture treatments received. Acupuncture effects were shown to favor females. The possible underlying mechanism may be due to different neural effects between males and females induced by acupuncture, which were proved in the functional magnetic resonance imaging (MRI) study [69].

Through subgroup analysis, we found that the benefits of acupuncture decrease as baseline comorbidities increase and with the older age. Since the elderly and patients with more medical conditions have a poorer physical constitution and organic function, the curative effect of acupuncture is not easily detected. In addition, patients with older age or complicated medical conditions are more susceptible to infections, which may worsen the outcomes of stroke prognosis. Therefore, traditional Chinese doctors may use other treatment methods, such as moxibustion, laser acupuncture, electroacupuncture, and infrared or far-infrared therapy, to help these patients. Moreover, in the theory of traditional Chinese medicine, acupuncture can regulate but not generate the qi of human meridian circulation [70]. With increased age, meridian qi gradually decreases after 40 years of age [71]. Consequently, elderly patients have little qi to be regulated and show a poorer response after acupuncture [71].

The risk of poststroke comorbidities exhibited a dose-dependent decrease as patients received more acupuncture treatments. Although 15 acupuncture treatments per month was the limit imposed by Taiwan's National Health Insurance System, there is no evidence indicating the optimal dosage of acupuncture for stroke patients. In traditional Chinese medicine theory, acupuncture may regulate yin and yang and modulate the body to become balanced [72]. Therefore, more treatments may help stroke patients balance their health and prevent poststroke comorbidities.

4.4. Limitations. The systematic review and meta-analysis of nationalized retrospective cohort studies have some limitations due to the database characteristics and study method. First, the studies used retrospective data claimed from NHIRD, which lacked information regarding possible confounding factors, such as disease severity, stroke lesion characteristics, family history, baseline lifestyle, physical status, psychiatric condition, and laboratory examinations. Second, although the accuracy of ICD-9-CM codes in the database has been proved in previous studies [2], the misdiagnosis that violates the validity of ICD cannot be avoided. Third, information on the actual acupuncture points and manipulations applied in treatment was absent. Diversified and individualized acupoints selection without adequately standardized protocols may have affected the accuracy of the estimated treatment effects. Fourth, since our review was based on observational retrospective cohort studies that are nonrandomized studies, we cannot deduce the cause-andeffect relationship between acupuncture and poststroke comorbidities regarding the significant association. Moreover, the level of evidence of retrospective cohort studies is lower than that of randomized controlled trials. Fifth, the population could be overlapped in several studies since they share the same database with the same inclusion criteria and ICD-9 diagnosis code; therefore, the efficacy of meta-analysis might be limited.

\section{Conclusions}

Our systematic review and meta-analysis of nationalized cohort studies revealed that acupuncture is associated with reduced incidence of poststroke comorbidities such as stroke recurrence, new-onset AMI, pneumonia, dementia, epilepsy, and UTI. The meta-analysis results of eight trials showed that acupuncture exerts a significant effect in reducing the risk of poststroke comorbidities compared to the nonacupuncture group with a hazard ratio: 0.776 (95\% CI: 0.719-0.838). Regarding subgroup analysis, HR results involving different age strata and a number of acupuncture treatments received also show a significant correlation after meta-analysis. However, eight included nationwide cohort studies were assessed as moderate to high risk of bias. As a result, further studies that evaluate adverse events and include a long-term follow-up should be conducted to 
determine the efficacy, safety, and side effects of acupuncture for poststroke comorbidities in real-world settings.

\section{Data Availability}

The data that support the findings of this study are available from the corresponding authors upon reasonable request.

\section{Conflicts of Interest}

The authors declare that there are no conflicts of interest regarding the publication of this paper.

\section{Authors' Contributions}

Li-Kung $\mathrm{Wu}$ and Chung-Shan Hung are the authors who contributed equally to this work.

\section{Supplementary Materials}

Table S1. Supporting data for Forest plot of Hazard Ratio (HR) of post-stroke comorbidities with acupuncture intervention compared with non-acupuncture control. A: acupuncture group, C: control group. Table S2. Supporting data extracted from each study showing HRs with 95\% confidence intervals of different age strata on post-stroke comorbidities after acupuncture treatment. (HR: hazard ratio; LCI: lower confidence intervals; UCI: upper confidence intervals). Table S3. Meta-analysis results of different age strata on post-stroke comorbidities after acupuncture treatment. (k: number of samples; I2: heterogeneity; $\mathrm{p}$ : $\mathrm{p}$ value). Table S4. Supporting data extracted from each study showing HRs with 95\% confidence intervals of various acupuncture courses on post-stroke comorbidities after acupuncture treatment. Table S5. Supporting data for Metaanalysis results of various acupuncture courses on poststroke comorbidities after acupuncture treatment. (k: number of samples; I2: heterogeneity; p: p-value). Figure S1. Forest plot of Hazard Ratio (HR) of post-stroke depression with acupuncture intervention compared with non-acupuncture control. (Supplementary Materials)

\section{References}

[1] G. A. Donnan, M. Fisher, M. Macleod, and S. M. Davis, "Stroke," Lancet, vol. 371, no. 9624, pp. 1612-1623, 2008.

[2] S.-W. Weng, T.-L. Chen, C.-C. Yeh et al., "An investigation of the use of acupuncture in stroke patients in Taiwan: a national cohort study," BMC Complementary and Alternative Medicine, vol. 16, no. 1, p. 321, 2016.

[3] Y. Cai, C. S. Zhang, S. Liu et al., "Electroacupuncture for poststroke spasticity: a systematic review and meta-analysis," Archives of Physical Medicine and Rehabilitation, vol. 98, no. 12, pp. 2578-2589, 2017.

[4] F. Liu, Z.-M. Li, Y.-J. Jiang, and L.-D. Chen, "A meta-analysis of acupuncture use in the treatment of cognitive impairment after stroke," Journal of Alternative \& Complementary Medicine, vol. 20, no. 7, pp. 535-544, 2014.

[5] J. Zhang, J. Chen, J. Chen et al., "Early filiform needle acupuncture for poststroke depression: a meta-analysis of 17 randomized controlled clinical trials," Neural Regeneration Research, vol. 9, no. 7, pp. 773-784, 2014.

[6] M. Ratmansky, A. Levy, A. Messinger, A. Birg, L. Front, and I. Treger, "The effects of acupuncture on cerebral blood flow in post-stroke patients: a randomized controlled trial," Journal of Alternative \& Complementary Medicine, vol. 22, no. 1, pp. 33-37, 2016.

[7] Y. M. Liu, X. J. Liu, S. S. Bai et al., "The effect of electroacupuncture on $\mathrm{T}$ cell responses in rats with experimental autoimmune encephalitis," Journal of Neuroimmunology, vol. 220, no. 1-2, pp. 25-33, 2010.

[8] J. Prosser, L. MacGregor, K. R. Lees, H.-C. Diener, W. Hacke, and S. Davis, "Predictors of early cardiac morbidity and mortality after ischemic stroke," Stroke, vol. 38, no. 8, pp. 2295-2302, 2007.

[9] M.-C. Feng, Y.-C. Lin, Y.-H. Chang et al., “The mortality and the risk of aspiration pneumonia related with dysphagia in stroke patients," Journal of Stroke and Cerebrovascular Diseases, vol. 28, no. 5, pp. 1381-1387, 2019.

[10] C. J. Winstein, J. Stein, R. Arena et al., "Guidelines for adult stroke rehabilitation and recovery: a guideline for healthcare professionals from the American heart association/American stroke association," Stroke, vol. 47, no. 6, pp. e98-e169, 2016.

[11] A. Yang, H. M. Wu, J. L. Tang, L. Xu, M. Yang, and G. J. Liu, "Acupuncture for stroke rehabilitation," Cochrane Database of Systematic Reviews, vol. 8, Article ID Cd004131, 2016.

[12] J. Park, A. R. White, M. A. James et al., "Acupuncture for subacute stroke rehabilitation," Archives of Internal Medicine, vol. 165, no. 17, pp. 2026-2031, 2005.

[13] H. M. Sterne Jac, B. C. Reeves, J. Savović et al., "ROBINS-I: a tool for assessing risk of bias in non-randomized studies of interventions," BMJ, vol. 355, Article ID i4919, 2016.

[14] T. J. Higgins JPT, J. Chandler, M. Cumpston, T. Li, M. J. Page, and V. A. Welch, Eds., Cochrane Handbook for Systematic Reviews of Interventions, Cochrane 2020, London, UK, 2020.

[15] L. A. McGuinness and J. P. T. Higgins, "Risk-of-bias VISualization (robvis): an R package and Shiny web app for visualizing risk-of-bias assessments," Research Synthesis Methods, vol. 12, no. 1, pp. 55-61, 2020.

[16] R Development Core Team, R.: A Language and Environment for Statistical Computing, $\mathrm{R}$ Foundation for Statistical Computing, Vienna, Austria, 2020.

[17] S. Balduzzi, G. Rücker, and G. Schwarzer, "How to perform a meta-analysis with R: a practical tutorial," Evidence-Based Mental Health, vol. 22, no. 4, pp. 153-160, 2019.

[18] J. P. T. Higgins and S. G. Thompson, "Quantifying heterogeneity in a meta-analysis," Statistics in Medicine, vol. 21, no. 11, pp. 1558-6715, 2002.

[19] J.-L. Yang, T.-L. Chen, C.-C. Yeh et al., "Acupuncture treatment and the risk of urinary tract infection in stroke patients: a nationwide matched cohort study," Acupuncture in Medicine, vol. 37, no. 3, pp. 175-183, 2019.

[20] C.-Y. Lu, H.-C. Huang, H.-H. Chang et al., "Acupuncture therapy and incidence of depression after stroke," Stroke, vol. 48, no. 6, pp. 1682-1684, 2017.

[21] C.-C. Chang, T.-L. Chen, C.-S. Lin et al., "Decreased risk of pneumonia in stroke patients receiving acupuncture: a nationwide matched-pair retrospective cohort study," PLoS One, vol. 13, no. 5, Article ID e0196094, 2018.

[22] S.-F. Chuang, C.-C. Shih, C.-C. Yeh et al., "Decreased risk of acute myocardial infarction in stroke patients receiving acupuncture treatment: a nationwide matched retrospective cohort study," BMC Complementary and Alternative Medicine, vol. 15, no. 1, p. 318, 2015. 
[23] C.-C. Shih, C.-C. Liao, M.-F. Sun et al., "A retrospective cohort study comparing stroke recurrence rate in ischemic stroke patients with and without acupuncture treatment," Medicine, vol. 94, no. 39, p. e1572, 2015.

[24] C.-C. Shih, C.-C. Yeh, C.-J. Hu et al., "Risk of dementia in patients with non-haemorrhagic stroke receiving acupuncture treatment: a nationwide matched cohort study from Taiwan's National Health Insurance Research Database," BMJ Open, vol. 7, no. 6, Article ID e013638, 2017.

[25] S. P. Tseng, Y. C. Hsu, C. J. Chiu, and S. T. Wu, "A populationbased cohort study on the ability of acupuncture to reduce post-stroke depression," Medicines (Basel, Switzerland), vol. 4, no. 1, 2017.

[26] S.-W. Weng, C.-C. Liao, C.-C. Yeh et al., "Risk of epilepsy in stroke patients receiving acupuncture treatment: a nationwide retrospective matched-cohort study," BMJ Open, vol. 6, no. 7, Article ID e010539, 2016.

[27] H. Cai, B. Ma, X. Gao, and H. Gao, "Tongue acupuncture in treatment of post-stroke dysphagia," International Journal of Clinical and Experimental Medicine, vol. 8, no. 8, pp. 14090-14094, 2015.

[28] K. T. Luo, F. Yang, X. D. Bian, Z. Y. Lou, and J. Ge, "Clinical study of acupuncture treatment for pulmonary infection after acute cerebral infarction," Shanghai Journal of Acupuncture and Moxibustion [Shang Hai Zhen Jiu Za Zhi], vol. 35, no. 9, pp. 1070-1072, 2016.

[29] Y. Liu, L. Liu, and X. Wang, "Electroacupuncture at points Baliao and Huiyang (BL35) for post-stroke detrusor overactivity," Neural Regeneration Research, vol. 8, no. 18, pp. 1663-1672, 2013.

[30] K. W. Yu, C. L. Lin, C. C. Hung et al., "Effects of electroacupuncture on recent stroke inpatients with incomplete bladder emptying: a preliminary study," Clinical Interventions in Aging, vol. 7, pp. 469-474, 2012.

[31] P.-F. Shen, L. Kong, and L.-W. Ni, "Acupuncture intervention in ischemic stroke: a randomized controlled prospective study," American Journal of Chinese Medicine, vol. 40, no. 4, pp. 685-693, 2012.

[32] S.-H. Lee and S. M. Lim, "Acupuncture for insomnia after stroke: a systematic review and meta-analysis," BMC Complementary and Alternative Medicine, vol. 16, no. 1, p. 228, 2016.

[33] Y. Liu, H. X. Zhang, and G. H. Chen, "Therapeutic effects of scalp-acupuncture in patients with vascular dementia induced by cerebral infarction: a randomized controlled trial," Journal of Chinese Integrative Medicine, vol. 6, no. 8, pp. 806-809, 2008.

[34] R. Liu, K. Zhang, Q.-Y. Tong, G.-W. Cui, W. Ma, and W.-D. Shen, "Acupuncture for post-stroke depression: a systematic review and meta-analysis," BMC Complementary Medicine and Therapies, vol. 21, no. 1, p. 109, 2021.

[35] W. Fan, X. Kuang, J. Hu et al., "Acupuncture therapy for poststroke spastic hemiplegia: a systematic review and metaanalysis of randomized controlled trials," Complementary Therapies in Clinical Practice, vol. 40, Article ID 101176, 2020.

[36] A. J. Liu, J. H. Li, H. Q. Li et al., "Electroacupuncture for acute ischemic stroke: a meta-analysis of randomized controlled trials," The American Journal of Chinese Medicine, vol. 43, no. 8, pp. 1541-1566, 2015.

[37] L. Chen, J. Fang, R. Ma et al., "Additional effects of acupuncture on early comprehensive rehabilitation in patients with mild to moderate acute ischemic stroke: a multicenter randomized controlled trial," BMC Complementary and Alternative Medicine, vol. 16, no. 1, p. 226, 2016.
[38] X. Yan, Y. Zhang, and Z. Liu, "Acupuncture for post-stroke spasticity: a systematic review of randomized controlled trial," Journal of Alternative \& Complementary Medicine, vol. 22, no. 6, p. A42, 2016.

[39] J. Zhan, R. Pan, M. Zhou et al., "Electroacupuncture as an adjunctive therapy for motor dysfunction in acute stroke survivors: a systematic review and meta-analyses," BMJ Open, vol. 8, no. 1, Article ID e017153, 2018.

[40] F. K.-h. Sze, E. Wong, K. K. H. Or, J. Lau, and J. Woo, "Does acupuncture improve motor recovery after stroke?" Stroke, vol. 33, no. 11, pp. 2604-2619, 2002.

[41] L. X. Li and K. Deng, "Acupuncture combined with swallowing training for poststroke dysphagia: a meta-analysis of randomised controlled trials," Acupuncture in Medicine, vol. 37, no. 2, pp. 81-90, 2019.

[42] C. Yu, B. Shen, and S. W. Xu, "Effect of needle retention time on fatigue of post ischemic stroke in patients," Journal of clinical acupuncture and moxibustion [Zhen Jiu Lin Chuang Za Zhi], vol. 31, no. 6, pp. 1-3, 2015.

[43] H.-Y. Liao, W.-C. Ho, C.-C. Chen et al., "Clinical evaluation of acupuncture as treatment for complications of cerebrovascular accidents: a randomized, sham-controlled, subject- and assessor-blind trial," Evidence-based Complementary and Alternative Medicine, vol. 2017, Article ID 7498763, 10 pages, 2017.

[44] M. Wang, F. Ma, and H. Chen, "Protective effects of acupuncture on brain tissue following ischemia/reperfusion injury," Neural Regeneration Research, vol. 3, no. 3, pp. 309-312, 2008.

[45] L. Lu, X.-G. Zhang, L. L. D. Zhong et al., "Acupuncture for neurogenesis in experimental ischemic stroke: a systematic review and meta-analysis," Scientific Reports, vol. 6, no. 1, Article ID 19521, 2016.

[46] C. Iadecola and J. Anrather, "The immunology of stroke: from mechanisms to translation," Nature Medicine, vol. 17, no. 7, pp. 796-808, 2011.

[47] Z. Wang, T. Chen, M. Long et al., "Electro-acupuncture at acupoint ST36 ameliorates inflammation and regulates Th1/ Th2 balance in delayed-type hypersensitivity," Inflammation, vol. 40, no. 2, pp. 422-434, 2017.

[48] J. Gui, F. Xiong, J. Li, and G. Huang, "Effects of acupuncture on Th1, th2 cytokines in rats of implantation failure," Evidence-Based Complementary and Alternative Medicine, vol. 2012, Article ID 893023, 10 pages, 2012.

[49] P. Kidd, "Th1/Th2 balance: the hypothesis, its limitations, and implications for health and disease," Alternative Medicine Review: A Journal of Clinical Therapeutic, vol. 8, no. 3, pp. 223-246, 2003.

[50] F. Wang, G. W. Cui, and L. Kuai, "Role of acupoint Area collagen fibers in anti-inflammation of acupuncture lifting and thrusting manipulation," Evidence-Based Complementary and Alternative Medicine, vol. 2017, Article ID 2813437, 8 pages, 2017.

[51] H.-D. Lim, M.-H. Kim, C.-Y. Lee, and U. Namgung, "Antiinflammatory effects of acupuncture stimulation via the vagus nerve," PLoS One, vol. 11, no. 3, Article ID e0151882, 2016.

[52] J. H. Wang, X. Z. Du, and T. Z. Zhang, "Scalp-acupuncture improves neurological function by regulating expression of IL-10 mRNA, IL-6 mRNA and TNF- $\alpha$ of parahippocampal gyrus in cerebral ischemic rats," Zhen Ci Yan Jiu, vol. 44, no. 3, pp. 183-188, 2019.

[53] Z. Wang, B. Nie, D. Li et al., "Effect of acupuncture in mild cognitive impairment and Alzheimer disease: a functional MRI study," PLoS One, vol. 7, no. 8, Article ID e42730, 2012. 
[54] K. Nishijo, H. Mori, K. Yosikawa, and K. Yazawa, "Decreased heart rate by acupuncture stimulation in humans via facilitation of cardiac vagal activity and suppression of cardiac sympathetic nerve," Neuroscience Letters, vol. 227, no. 3, pp. 165-168, 1997.

[55] H.-S. Byeon, S.-K. Moon, S.-U. Park et al., "Effects of GV20 acupuncture on cerebral blood flow velocity of middle cerebral artery and anterior cerebral artery territories, and CO2 reactivity during hypocapnia in normal subjects," Journal of Alternative \& Complementary Medicine, vol. 17, no. 3, pp. 219-224, 2011.

[56] M. Tsuchiya, E. F. Sato, M. Inoue, and A. Asada, "Acupuncture enhances generation of nitric oxide and increases local circulation," Anesthesia \& Analgesia, vol. 104, no. 2, pp. 301-307, 2007.

[57] Y. Du, L. Shi, J. Li, J. Xiong, B. Li, and X. Fan, “Angiogenesis and improved cerebral blood flow in the ischemic boundary area were detected after electroacupuncture treatment to rats with ischemic stroke," Neurological Research, vol. 33, no. 1, pp. 101-107, 2011.

[58] F. A. Flachskampf, J. Gallasch, O. Gefeller et al., "Randomized trial of acupuncture to lower blood pressure," Circulation, vol. 115, no. 24, pp. 3121-3129, 2007.

[59] V. Aboyans and M. H. Criqui, "Can we improve cardiovascular risk prediction beyond risk equations in the physician's office?" Journal of Clinical Epidemiology, vol. 59, no. 6, pp. 547-558, 2006.

[60] M.-J. Lee, J.-S. Ryu, S.-K. Won et al., "Effects of acupuncture on chronic stress-induced depression-like behavior and its central neural mechanism," Frontiers in Psychology, vol. 10, p. 1353, 2019.

[61] A. J. Vickers and K. Linde, "Acupuncture for chronic pain," JAMA, vol. 311, no. 9, pp. 955-956, 2014.

[62] M. T. Cabioğlu and N. Ergene, "Electroacupuncture therapy for weight loss reduces serum total cholesterol, triglycerides, and LDL cholesterol levels in obese women," The American Journal of Chinese Medicine, vol. 33, no. 4, pp. 525-533, 2005.

[63] M. Miller, N. J. Stone, C. Ballantyne et al., "Triglycerides and cardiovascular disease," Circulation, vol. 123, no. 20, pp. 2292-2333, 2011.

[64] B.-R. Kim, J. H. Lim, S. A. Lee et al., "The relation between postvoid residual and occurrence of urinary tract infection after stroke in rehabilitation unit," Annals of Rehabilitation Medicine, vol. 36, no. 2, pp. 248-253, 2012.

[65] J. C. Kong, M. S. Lee, B.-C. Shin, Y.-S. Song, and E. Ernst, "Acupuncture for functional recovery after stroke: a systematic review of sham-controlled randomized clinical trials," Canadian Medical Association Journal, vol. 182, no. 16, pp. 1723-1729, 2010.

[66] P. Wu, E. Mills, D. Moher, and D. Seely, "Acupuncture in poststroke rehabilitation: a systematic review and metaanalysis of randomized trials," Stroke, vol. 41, no. 4, pp. e171-9, 2010.

[67] T. A. Lakka, J. M. Venalainen, R. Rauramaa, R. Salonen, J. Tuomilehto, and J. T. Salonen, "Relation of leisure-time physical activity and cardiorespiratory fitness to the risk of acute myocardial infarction in men," New England Journal of Medicine, vol. 330, no. 22, pp. 1549-1554, 1994.

[68] N. F. Gordon, M. Gulanick, F. Costa et al., "Physical activity and exercise recommendations for stroke survivors," Circulation, vol. 109, no. 16, pp. 2031-2041, 2004.

[69] A. Li, Y. H. Wang, and F. Zhang, "Acupuncture for gender differences and similarities in cerebral activity of health volunteers: a pilot fMRI study," Medicine (Baltimore), vol. 97, no. 50, Article ID e13655, 2018.

[70] P. Rong, B. Zhu, Y. Li et al., "Mechanism of acupuncture regulating visceral sensation and mobility," Frontiers of Medicine, vol. 5, no. 2, pp. 151-156, 2011.

[71] W. Bing, Yellow Emperor's Canon of Internal Medicine, China Ccience \& Technology Press, China, 2005.

[72] Y. Lee, S. Kim, T. Son, D. Kang, and B. Jung, "Meridian electrical potential response to acupuncture stimulation between operator and subject," Journal of Acupuncture and Meridian Studies, vol. 3, no. 4, pp. 249-254, 2010. 